\title{
Stemming influenza viruses
}

vaccination
strategies
that elicit
antibodies
directed
against the
stem region
of HA are
protective
against
multiple
influenza
strains

trimerization and exposure of the stem region that is recognized by bnAbs. The resulting 'H1 HA stabilized-stem proteins' (HA-SSs) were further fused to the ferritin subunit from Helicobacter pylori to create self-assembling nanoparticles (termed 'H1-SS-nps'), a strategy that has been shown to increase the ability of HA to elicit antibodies. Impagliazzo et al. used a different structure-based strategy to generate 'stable HA stem antigens' ('mini-HAs'); in a five-step process, they started with $\mathrm{H} 1 \mathrm{HA}$ proteins and generated soluble and stable HA monomers that lack the HA head group, maximized exposure of the stem region that is recognized by bnAbs, and then engineered the stem monomers to create stable trimeric mini-HAs.

Yassine et al. tested the protective efficacy of H1-SS-nps in mice and ferrets. Immunization with these nanoparticles elicited broad antibody responses against group 1 HA subtypes (including H1, H2, H5 and H9) in both mice and ferrets, whereas antibody responses against group 2 HA subtypes (H3 and H7) were observed in mice. Furthermore, mice and ferrets immunized with H1-SS-nps were protected against lethal infection with a heterosubtypic $\mathrm{H} 5 \mathrm{~N} 1$ virus; whereas all animals immunized with empty nanoparticles died, all mice and four out of six ferrets immunized with H1-SS-nps survived. Interestingly, the antibodydependent protection elicited by the H1-SS-np vaccine seemed to be mediated by effector mechanisms other than viral neutralization.

Impagliazzo et al. tested their mini-HAs for the ability to confer protection in mice and in nonhuman primates. In mice, immunization with the mini-HAs induced the generation of antibodies against group 1 (including $\mathrm{H} 1, \mathrm{H} 5$ and $\mathrm{H} 9$ ) and group 2 (including $\mathrm{H} 3$ and $\mathrm{H} 7$ ) virus strains. Furthermore, immunization with mini-HAs provided heterosubtypic protection against lethal infection with an $\mathrm{H} 5 \mathrm{~N} 1$ virus in all of the vaccinated mice. In cynomolgus monkeys, animals vaccinated with mini-HAs had reduced fever after infection with a heterologous H1N1 virus compared with mock-vaccinated monkeys, which was similar to what was observed in monkeys that had received the seasonal flu vaccine. However, vaccination with mini-HAs induced the generation of high titres of antibodies with the ability to neutralize $\mathrm{H} 5 \mathrm{~N} 1$, whereas vaccination with the seasonal flu vaccine did not.

Finally, both studies confirmed the key role of antibodies in mediating heterosubtypic protection by transferring immunoglobulin from mice immunized with either H1-SSnps or H1 mini-HAs, followed by infection with an $\mathrm{H} 5 \mathrm{~N} 1$ virus. In both cases, mice receiving the immunoglobulin from the immunized mice survived the challenge with a lethal infectious dose, whereas mice that received control immunoglobulin succumbed to infection.

Collectively, these studies demonstrate that vaccination strategies that elicit antibodies directed against the stem region of HA are protective against multiple influenza strains and pave the way for the development of a universal flu vaccine.

Cláudio Nunes-Alves

ORIGINAL RESEARCH PAPERS Impagliazzo, A. et al. A stable trimeric influenza hemagglutinin stem as a broadly protective immunogen. Science http://dx.doi.org/10.1126/science.aac7263 (2015) | Yassine, H. M. et al. Hemagglutinin-stem nanoparticles generate heterosubtypic influenza protection. Nat. Med. http://dx.doi.org/10.1038/ nm.3927 (2015) 